\title{
Modeling phase change materials behaviour in building applications: selected comments
}

\author{
Yvan Dutil $^{1}$,*, Daniel Rousse ${ }^{1}$, Stéphane Lassue $^{2}$, Laurent Zalewski $^{2}$, Annabelle Joulin $^{2}$, \\ Joseph Virgone ${ }^{3,5}$, Frédéric Kuznik ${ }^{4,5}$, Kevyn Johannes ${ }^{3,5}$, Jean-Pierre Dumas ${ }^{6}$, Jean-Pierre \\ Bédécarrats $^{6}$, Albert Castell ${ }^{7}$, Luisa F. Cabeza ${ }^{7}$ \\ 1 t3e Industrial research chair, École de technologie supérieure, Montréal, Canada \\ 2 Univ. Lille Nord de France, F-59000 Lille, France ; Univ. Artois, LGCgE, F-62400 Béthune, France \\ 3 Université de Lyon, CNRS, UMR5008, F-69622 Villeurbanne, France \\ 4 INSA-Lyon, CETHIL, F-69621 Villeurbanne, France \\ 5Université Lyon 1, CETHIL, F-69622 Villeurbanne, France \\ 6 LATEP, Université de Pau et des Pays de l'Adour, Pau, France \\ 7 GREA Innovació Concurrent,Universitat de Lleida, Lleida, Spain \\ * Corresponding author. Tel: +1 (418) 653-2910, Fax: +1 (514) 396-8950, E-mail: yvan@t3e.info
}

\begin{abstract}
In a recent meeting of IEA's Annex 23, several members presented their conclusions on the modeling of phase change materials behavior in the context of building applications. These conclusions were in agreement with those of a vast review, involving the survey of more than 250 journal papers, undertaken earlier by the group of École de technologie supérieure. In brief, it can be stated that, at this point, the confidence in reviewed models is too low to use them to predict the future behavior of a building with confidence. Moreover, it was found that overall thermal behaviors of PCM are poorly known, which by itself creates an intrinsic unknown in any model. Models themselves are most of time suspicious as they are often not tested in a very stringent or exhaustive way. In addition, it also appears that modeling parameters are somewhat too simplified to realistically describe the complete physics needed to predict the real life performance of PCMs added to a building. As a result, steps are now taken to create standard model benchmarks that will improve the confidence of the users. Hopefully, following these efforts, confidence will increase and usage of PCM in buildings should be eased.
\end{abstract}

Keywords: Phase change material, PCM characterization, Mathematical model, Model validation

\section{Context}

The ever increasing level of greenhouse gas emissions combined with the overall rise in fuel prices (although fluctuations occur) are today's main reasons behind efforts devoted to improve the use of various sources of energy. Economists, scientists, and engineers throughout the world are nowadays in search of: 1) strategies to reduce the demand;2) methods to ensure the security of the supplies; 3) technologies to increase the energy efficiency of power systems; and 4) new and renewable sources of energy to replace the limited and harmful fossil fuels.

One of the options to improve energy efficiency is to develop energy storage devices and systems in order to reduce the mismatch between supply and demand. In this context, latent heat storage could be considered. Indeed, it is particularly attractive since it provides a highenergy storage density and has the capacity to store energy at a constant temperature - or over a limited range of temperature variation - which is the temperature that corresponds to the phase transition temperature of the material. For instance, it takes 80 times as much energy to melt a given mass of water (ice) than to raise the same amount of water by $1^{\circ} \mathrm{C}$. For the interested reader, excellent global reviews that pertain to phase change materials and their various applications were proposed by Zalba et al. [1], Farid et al. [2], Zhang at al. [3], Tyagi and Buddhi [4], Regin et al. [5], Mondal [6], Mehling \& Cabeza [7], Sethi \& Sharma [8], Verma et al. [9], Sharma et al. [10], Dutil et al. [11] and Cabeza et al. [12]. 


\section{Modeling in building applications}

A better management of the fluctuations of the external temperatures, wind, solar load, and heating or cooling needs is possible by the use of phase change materials. In building applications, these materials undergo a phase change close to the desired room temperature, which allow storing a large amount of heat in a relatively small volume compared to liquid water, brick or concrete. This results in direct energy savings as the solar gains can be used when needed, thus reducing the energy consumption for heating in the winter and cooling in the summer. Moreover, in many countries, these materials could also be used to reduce the peak consumption leading to money savings in this particular case.

Nevertheless, high fidelity models are needed to guide the decisions of the architects and/or HVAC engineers in choosing optimum designs. Unfortunately, to formulate, implement, and validate such models is a rather difficult task mainly due to the non-linear nature of the problem. In addition, other technical issues add complexity to this problem. Here, we will discuss two of the most significant problems that should be addressed by the scientific community: phase change material characterization and model validation [11].

\subsection{PCM characterization}

The first problem faced even before beginning the modeling process is the characterization of the phase change materials (PCMs) themselves. In building applications, composite PCMs are the favored packaging method for inner walls applications. In this form, PCMs can be integrated into a building using the same techniques used for gypsum panel, which would provide a seamless integration. However, this type of material is rather difficult to characterize.

The key problem comes from the interaction with the substrate and the PCM in confined pores. This interaction affects both the melting and freezing temperatures as their respective enthalpy. To our knowledge, this phenomenon was first observed in building application by Hawes et al. [13], when they noted a drift in thermal properties of a PCM laced concrete over time. They attributed this effect to a migration of PCM in smaller pores. Their interpretation was supported by a previous work of Harnik et al. [14] on icing behavior of concrete.

Many physical models have been proposed to explain this behavior [15-25]. The thermodynamic properties of PCM composites are related in complex way to the size of the pores and to the chemical properties of the matrix and of the PCM. Mechanical confinement shifts the phase transition to higher temperatures due to increased pressure. Chemical interaction including dissolution between the compounds can shift up or down the melting/freezing temperature. The stochastic nature of the nucleation process means that supercooling is favored in small volumes. In consequence, melting and freezing phase change occurs at different temperatures. The phase change range is also broadened. This has the practical consequence that it is necessary to measure both melting and freezing curves and this over a wide range of temperature.

Even then, adequate characterization of PCM is a d ifficult task. For example, we have observed that reported enthalpy of melting and freezing can differ by more than as $15 \%$ in composite PCMs (ex: construction material [26-28] and polymer [29-31]). This is obviously unphysical since conservation of energy imposes that both values should be equal if the energy is stored in the PCM. Still, there is a possibility that some energy might be stored mechanically by the deformation of the matrix as a consequence of the PCM dilatation. To 
our knowledge, this hypothesis has never been tested. While, if proven true, this phenomenon might provide new approaches to fine tune composite PCM thermal behavior.

In practice, the broad width of the composite PCM freezing/melting curve impairs the separation between latent and sensible heat. In addition, in some cases, there is an indication in many published measurements that at least a part of the PCM stays in supercooling state during the whole thermal cycle. In addition, heat capacity value and conductivity are different between liquid and solid phases. All these problems make very difficult to define a meaningful baseline to extract the latent heat curve.

In addition, hysteresis in the cooling/heating curve has been observed [32-36]. This behavior is not fully explained but is likely to be related to a complex interaction between the stochastic nature of the nucleation process (heterogeneous or homogeneous), progressive dissolution, glass transition or metastable crystalline phases. This has for consequence that each DSC curve is dependent on the history of temperature and its rate of change. Measurement procedures for this effect are still in development.

In general, thermophysical properties measurements are done on a small sample. However, due to the non classical behavior of composite PCM, it is unclear whether these measurements are representative of the macroscopic thermal properties of the material. A more detailed study is under investigation, which consists on the consideration of the heat transfers within the calorimeter cells. The goal is to determine the true value of specific enthalpy regardless of experimental conditions (sample mass, heating and cooling rates) [38]. This method also allows the determination of thermal properties by inverse methods [39]. In addition, potential drift in the thermophysical properties overtime are not always taken into account in the experimental protocol. Through a literature review, we have observed that tests of the stability of PCM composite extend from a few cycles to 5000 thermal cycles! Since, for building applications, lifetime of components are decades long the latter value is certainly more suitable.

In conclusion, improved thermal characterisation procedures are needed and will be certainly welcomed by modellers.

\subsection{Model validation}

The validation of modelling algorithms is also troublesome. While not restricted to building applications [11], it is more critical in this case due to the relatively small temperature changes involved in a typical building application. In surveyed papers on modeling, all older models for PCMs behavior had experimental counterparts to validate the modeling of the problem. This was done to adequately validate the appropriateness of the set of equations and that of the subsequent formulation of a numerical method to solve the relevant sets of discretized equations. Many of these early studies also involved analytical solutions used to validate the model for selected problems that admit closed form solutions [11].

However, as time went by, the authors relied more and more on ot her studies, mostly numerical ones, to validate their own numerical results. Many of the recent studies discuss their results qualitatively only, as the comparison with a graph taken from a publication may be somehow hazardous. And, interestingly, among the numerous - more than 250 references and studies reported in [11], in only one the authors stated that the results were not "in good agreement with those found in the references". In recent studies, the proportion of 
analyses which rely on commercial codes increases and the discussions that pertain to stability, convergence, grid independence and other related numerical issues decrease.

Statements are almost never made on the agreement or disagreement with previous results. This may be explained partly by the engineering scientific culture, where challenging or trying to duplicate previous works is not a common practice. As an illustration of this observation, we noticed that the work of Heim and Clark [40-41] predicts accumulation of heat on a seasonal basis. This is certainly an extraordinary claim that would open door to new applications. Nevertheless, up to now, nobody has either duplicated or refutated it.

However, engineering sociology merely reflects the practical constraint of doing such crossvalidations. Materials, geometries, testing conditions and models are almost always different from one study to another. In such conditions, even for the most dedicated researcher, it is very hard to validate previous work. In our mind, this is a serious issue. Without a common ruler, it is impossible to formulate a meaningful recommendation about a technology.

Finally, we found that there is little comparison between various models and experiments. Every research group seems to have its own numerical model. To our knowledge, all these models were claimed to work well. Nevertheless, recent works [32-37] indicate that the presence of hysteresis creates some problem in the modeling itself since the thermal behavior of the PCM will depend on the history of heat loading. At this moment, solution to this problem is an open question. While this is a very new concern and might not be that important, this raises some doubts on previous results.

\subsection{Further steps}

To address some of these problems, the IEA annex 23 has prepared two standard cases to test numerical models [29-30]. The first of these tests was a simple unidirectional wall, with inclusion of a classical phase change material within the wall. Three teams developed a model for this case. While two of those models were closely predicting the same results, a third model presented a significant discrepancy with the other two. At this moment, not enough models are available to find the root cause of the observed difference. The main suspect is a small variation in the numerical description of the latent energy curve.

The existence of such divergence with a simple situation is by itself a strong warning about the models reliability. A second benchmark is now proposed. This benchmark is based on a small cubicle using PCM in its walls. In that case, high quality experimental data are used as a reference. To populate a database of benchmark, members of the annex 23 are invited to submit there own experimental data.

These initiatives are certainly a step in the right direction. Their use as a validation tool should be considered by any researchers working into application of PCM in building. Nevertheless, results are too fragmentary at this point to produce general guideline for researchers.

\section{Conclusion}

While the applications of PCM in building are promising as a tool to reduce energy consumption, there are still many roadblocks on the widespread utilization. To optimize their utilization in buildings, reliable models are needed. At this point, the confidence in models is too low to be use to predict the future behavior of a building. However, thermal behavior of PCM themselves are poorly known, which by itself create a huge unknown in model. Models themselves are suspicious as they are rarely tested in a very stringent way. 
In addition, it also appears to us that modeling parameters are somewhat too simplified to realistically describe the real life performance of PCM addition into buildings. For example, seldom complete meteorological information (solar irradiation, external temperature and wind) are used as inputs. However, correlation and anti-correlation between these factors could strongly affect the results. In addition, in most systems modeled, thermal loads are restricted to solar heating. Additional heat from appliances will certainly affect the results. Also most of the time modeling is done on individual rooms or few rooms aligned in a perfect east-west alignment and empty. In real life, most houses are not perfectly oriented, have additional room with little solar heating, are equipped with furniture, and are occupied by people. This will both modify the thermal loading and the effective storage mass of the building. From our analysis of the literature, typical gain in energy efficiency by the utilization of PCM is expected to be roughly about $10-15 \%$. In consequence, the factors not included in models could easily change the overall conclusion about the pertinence of PCM in building application.

The steps taken now by the IEA ECES IA Annex 23 to create standard model benchmark will improve the confidence of the users. Phase change material characterization is still an unresolved issue, but many research teams work on it. Hopefully, following these efforts, confidence will increase and usage of PCM in building will be more straightforward.

\section{Acknowledgements}

This work was supported by the t3e industrial research chair and its financial partners. The authors would like to acknowledge their invaluable contributions. The work was partially funded by the European Union (COST Action COST TU0802) and the Spanish Government (project ENE2008-06687-C02-01/CON). The authors would like to thank the Catalan Government for the quality accreditation given to their research group (2009 SGR 534).

\section{References}

[1] B. Zalba, J.M. Marín, L.F. Cabeza, H. Mehling, Review on thermal energy storage with phase change: materials, heat transfer analysis and applications, Applied Thermal Engineering 23, 2003, pp. 251-283.

[2] M. M. Farid, A. M. Khudhair, S. A. K. Razack, and S. Al-Hallaj, A review on ph ase change energy storage: materials and applications, Energy Conversion and Management, vol. 45 (9-10), 2004, pp.1597-1615

[3] Y. Zhang, G. Zhou, K. Lin, Q. Zhang, and H. Di , A pplication of latent heat thermal energy storage in buildings: State-of-the-art and outlook, Building and Environment, vol. 42(6), 2007, pp. 2197-2209

[4] V. V. Tyagi, and D. Buddhi, PCM thermal storage in buildings: A state of art, Renewable and Sustainable Energy Reviews, vol. 11(6), 2007, pp. 1146-1166

[5] A. F. Regin, S.C. Solanki, and J.S. Saini, Heat transfer characteristics of thermal energy storage system using PCM capsules: A review, Renewable and Sustainable Energy Reviews, vol. 12(9), 2008, pp. 2438-2458

[6] S. Mondal, Phase change materials for smart textiles - An overview, Applied Thermal Engineering, vol. 28(11-12), 2008, pp. 1536-1550

[7] H. Mehling, L.F. Cabeza. Heat and cold storage with PCM. An up to date introduction into basics and applications. Berlin, Springer, 2008. ISBN: 978-3-540-68557-9 
[8] V.P. Sethi, and S.K. Sharma, Survey and evaluation of heating technologies for worldwide agricultural greenhouse applications, Solar Energy, vol. 82(9), 2008, pp. 832 8598

[9] P. Verma, Varun, and S.K. Singal, Review of mathematical modeling on 1 atent heat thermal energy storage systems using phase-change material, Renewable and Sustainable Energy Reviews, vol. 12(4), 2008, pp.999-1031

[10]A. Sharma et al., Review on thermal energy storage with phase change materials and applications, Renewable and Sustainable Energy Review, Volume 13 (2), Issue 2, 2009, pp. $318-345$

[11]Y. Dutil, D. R. Rousse, N. Ben Salah, S. Lassue, L. Zalewski A review on phase-change materials: Mathematical modeling and simulations, Renewable and Sustainable Energy Reviews 15, Issue 1, 2011, pp. 112-130

[12]L.F. Cabeza, A. Castell, C. Barreneche, A. de Gracia, A. I. Fernández, Materials used as PCM in thermal energy storage in buildings: A review, Renewable and Sustainable Energy Reviews, 2011, doi: 10.1016/j.rser.2010.11.018.

[13]D.W. Hawes, D. Banu, D. Feldman, The stability of phase change materials in concrete, Solar Energy Materials and Solar Cells 27, 1992, pp. 103-118

[14]A.B. Harnik, V. Meier and A. Rosli, in: Combined Influence of Freezing and Deicing Salt on Concrete - Physical Aspects, Durability of Building Materials and Components, ASTM STP 691, Eds. P.J. Sereda and G.G. Litvan, ASTM, 1980, pp. 476-483.

[15]J. P. Bédécarrats, F. Strub, B. Falcon and J. P. Dumas, Phase-change thermal energy storage using spherical capsules: performance of a test plant, Int J. Refrig. Vol. 19, No. 3, 1996, pp. 187-196

[16]R. Radhakrishnan, K.E. Gubbins, Free energy studies of freezing in slit pores: an orderparameter approach using Monte Carlo simulation, Mol. Phys. 96, 1999, pp. 1249-1267

[17]R. Radhakrishnan, K.E Gubbins, K., Watanabe, K., Kaneko, Freezing of simple fluids in microporous activated carbon fibers: comparison of simulation and experiment. Journal of Chemical Physics 111, 1999, pp. 9058-9067

[18]G. H. Findenegg, A. Schreiber, Freezing and melting of water in ordered nanoporous silica materials. In: Setzer, M.J., Auberg, R., Keck, H.J. (Eds.), Proceedings of the International RILEM Workshop on Frost Resistance of Concrete, Cachan Cedex, France, 2002, pp. 105-116

[19]Y. Cai et al, Preparation and characterizations of HDPE-EVA alloy/OMT nanocomposites/paraffin compounds as a shape stabilized phase change thermal energy storage material, Thermochimica Acta 451, 2006, pp. 44-51

[20]Q. Cao, P. Liu, Hyperbranched polyurethane as novel solid-solid phase change material for thermal energy storage, European Polymer Journal 42, 2006, pp. 2931-2939

[21]Q. Cao, P. Liu, Crystalline-amorphous phase transition of hyperbranched polyurethane phase change materials for energy storage, J Mater Sci 42, 2007, pp. 5661-5665

[22]D. Zhang, K. Wu, Z., Li, Tuning effect of porous media's structure on the phase change behaviour of organic phase change matters. Journal of Tongji University 32, 2004, pp . $1163-1167$

[23]D. Zhang, J. Zhou, K. Wu, Z. Li, Granular phase changing composites for thermal energy storage, Solar Energy 78, 2005, pp. 471-480 
[24]D. Zhang, S. Tian, D. Xiao, Experimental study on the phase change behavior of phase change material confined in pores, Sol. Energy 81, 2007, pp. 653-660

[25]Y. Yamagishi, T. Sugeno, T. H. Takeuci-II, A. T. Pyatenko, An evaluation of microencapsulated PCM for use in cold energy transportation medium, Energy Conversion Engineering Conference, 1996, pp. 2077 - 2083

[26]A. Karaipekli, A. Sar1, Capric-myristic acid/vermiculite composite as form-stable phase change material for thermal energy storage, Solar Energy 83, 2009, pp. 323-332

[27] W. Wang, X.Yang, Y. Fang, J. Ding, J. Yan, Enhanced thermal conductivity and thermal performance of form-stable composite phase change materials by using b-Aluminum nitride, Applied Energy 86, 2009, pp. 1196-1200

[28]G. Fang,H. Li, X. Liu, Preparation and properties of lauric acid/silicon dioxide composites as form-stable phase change materials for thermal energy storage, Materials Chemistry and Physics 122 , 2010, pp. 533-536

[29]I. Krupa, A.S. Luyt, Thermal properties of uncross-linked and cross-linked LLDPE/wax blends, Polymer Degradation and Stability 70, 2000, pp. 111-117

[30]H.S. Mpanza, A.S. Luyt, Comparison of different waxes as processing agents for lowdensity polyethylene, Polymer Testing 25, 2006, pp. 436-442

[31]M. You, X. Wang, X. Zhang \& W. Li, Effects of Microencapsulated Phase Change Materials Granularity and Heat Treat Treatment Condition on the Structure and Performance of Polyurethane Foams, Modern Applied Science, vol2, number 4, 2008

[32] Kuznik, F., Virgone, J. Experimental investigation of wallboard containing phase change material: Data for validation of numerical modeling, Energy and Buildings 41, 2009, pp. $561-570$

[33]Kuznik, F., Virgone, J., Experimental assessment of a phase change material for wall building use, Applied Energy 86, 2009, pp. 2038-2046

[34]Diaconu, B. M., Cruceru, M., Novel concept of composite phase change material wall system for year-round thermal energy savings, Energy and Buildings 42, 2010, pp. 17591772

[35]B. M. Diaconu, S. Varga, A. C. Oliveira, Experimental assessment of heat storage properties and heat transfer characteristics of a phase change material slurry for air conditioning applications, Applied Energy 87, Issue 2, 2010, pp. 620-628

[36]Z. Younsi, L. Zalewski, S. Lassue, D. R. Rousse, A. Joulin, A Novel Technique for Experimental Thermophysical Characterization of Phase-Change Materials, International Journal of Thermophysics, Online First ${ }^{\mathrm{TM}}$, 28 December 2010

[37]A. Joulin, Z. Younsi, L. Zalewski, D. Rousse, S. Lassue, A numerical study of the melting of phase change material heated from a vertical wall of a rectangular enclosure, Int. Journal of Computational Fluid Dynamics, Vol. 23, No. 7, 2009, pp. 553-566

[38] T. Kousksou, A. Jamil, Y. Zeraouli, J.P. Dumas, Experimental and Modeling Study of Ice Melting, Journal of Thermal Analysis and Calorimetry 89, 1, 2007, pp. 31-36<

[39]Program MICMCP of the French National Research Agency (Stock-E) [laboratories: LaTEP, LGCgE, CETHIL]

[40]D. Heim, J.A. Clarke, Numerical modelling and thermal simulation of PCM- gypsum composites with ESP-r. Energy and Buildings, 36, 8, 2004, pp. 795-805 
[41]D. Heim, Isothermal storage of solar energy in building construction, Renewable Energy 35,2010 , pp. 788-796

[42]K. Johannes, J. Virgone, F. Kuznik, X. Wang, T. Haavi, One dimensional Benchmark based on P CM, IEA, Annex 23, A pplying Energy Storage in Buildings of the Future, 2010 